\title{
Interventions to Improve Follow-Up of Laboratory Test Results Pending at Discharge: A Systematic Review
}

\author{
Nedra S. Whitehead, MS, PhD¹, Laurina Williams, PhD, MPH ${ }^{2 *}$, Sreelatha Meleth, PhD¹, Sara Kennedy, MPH¹, \\ Paul Epner, $\mathrm{MS}^{3}$, Hardeep Singh, MD, MPH${ }^{4}$, Kathleene Wooldridge, MD, MPH${ }^{5}$, Anuj K. Dalal, MD, FHM' \\ Stacy E. Walz, PhD, MS, MT (ASCP)7, Tom Lorey, MD ${ }^{8}$, Mark L. Graber, MD
}

\begin{abstract}
${ }^{1} R T I$ International, Research Triangle Park, North Carolina; ${ }^{2}$ Centers for Disease Control and Prevention, Atlanta, Georgia; ${ }^{3}$ Paul Epner, LLC, Evanston, Illinois; ${ }^{4}$ Center for Innovations in Quality, Effectiveness and Safety, Michael E. DeBakey Veterans Affairs Medical Center, and Baylor College of Medicine, Houston, Texas; ${ }^{5}$ Vanderbilt University Medical Center, Nashville, Tennessee; ' ${ }^{6}$ Brigham and Women's Hospital, Harvard Medical School, Boston, Massachusetts; ${ }^{7}$ Arkansas State University, Jonesboro, Arkansas; ${ }^{8}$ Kaiser Permanente Northern California, Berkeley, California.
\end{abstract}

Failure to follow up test results pending at discharge (TPAD) from hospitals or emergency departments is a major patient safety concern. The purpose of this review is to systematically evaluate the effectiveness of interventions to improve follow-up of laboratory TPAD.

We conducted literature searches in PubMed, CINAHL, Cochrane, and EMBASE using search terms for relevant health care settings, transition of patient care, laboratory tests, communication, and pending or missed tests.

We solicited unpublished studies from the clinical laboratory community and excluded articles that did not address transitions between settings, did not include an intervention, or were not related to laboratory TPAD. We also excluded letters, editorials, commentaries, abstracts, case reports, and case series.

Of the 9,592 abstracts retrieved, eight met the inclusion criteria and reported the successful communication of TPAD. A team member abstracted predetermined data elements from each study, and a senior scientist reviewed the abstraction. Two experienced reviewers independently appraised the quality of each study using published Laboratory Medicine Best Practices (LMBPTM) A-6 scoring criteria.

We assessed the body of evidence using the A-6 methodology, and the evidence suggested that electronic tools or one-on-one education increased documentation of pending tests in discharge summaries. We also found that automated notifications improved awareness of TPAD.

The interventions were supported by suggestive evidence; this type of evidence is below the level of evidence required for $L M B P T M$ recommendations. We encourage additional research into the impact of these interventions on key processes and health outcomes. Journal of Hospital Medicine. 2018:13:631-636. Published online first February 27, 2018. (C) 2018 Society of Hospital Medicine

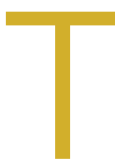

he 2015 National Academy of Sciences (NAS; formerly the Institute of Medicine [IOM]) report, Improving Diagnosis in Health Care, attributes up to $10 \%$ of patient deaths and $17 \%$ of hospital adverse events to diagnostic errors, ${ }^{1}$ one cause of which is absent or delayed follow-up of laboratory test results. ${ }^{2}$ Poor communication or follow-up of laboratory tests with abnormal results has been cited repeatedly as a threat to patient safety. ${ }^{1,3,4}$ In a survey of internists, $83 \%$ reported at least one unacceptably delayed laboratory test result during the previous 2 months. ${ }^{5}$

\footnotetext{
*Author for correspondence: Laurina Williams, PhD, MPH, Centers for Disease Control and Prevention, Center for Surveillance, Epidemiology, and Laboratory Services, Division of Laboratory Systems, 1600 Clifton Road, NE, MS G25, Atlanta, GA 30329; Telephone: 404-498-2267; Fax: 404-498-2215 E-mail: low1@cdc.gov

Additional Supporting Information may be found in the online version of this article.
}

Received: June 30, 2017; Revised: November 29, 2017;

Accepted: December 17, 2017

๑ 2018 Society of Hospital Medicine DOI 10.12788/jhm.2944
Care transitions magnify the risk of missed test results. ${ }^{6,7} \mathrm{Up}$ to $16 \%$ of all emergency department (ED) and $23 \%$ of all hospitalized patients will have pending laboratory test results at release or discharge. ${ }^{6}$ The percentage of tests that received follow-up ranged from $1 \%$ to $75 \%$ for tests done in the ED and from $20 \%$ to $69 \%$ for tests ordered on inpatients. In one study, $41 \%$ of all surveyed medical inpatients had at least one test result pending at discharge (TPAD). When further studied, over $40 \%$ of the results were abnormal and $9 \%$ required action, but the responsible physicians were unaware of $62 \%$ of the test results. ${ }^{8}$ Many examples of morbidity from such failure have been reported. One of many described by El-Kareh et al., for example, is that of an 81-year-old man on total parenteral nutrition who was treated for suspected line infection and discharged without antibiotics, but whose blood cultures grew Klebsiella pneumoniae after his discharge. 9 Another example, presented on the Agency for Healthcare Research and Quality (AHRQ) Patient Safety Network, reported a patient admitted for a urinary tract infection and then discharged from the hospital on trimethoprim-sulfamethoxazole. He returned to the hospital 11 days later with severe sepsis. Upon review, the urine culture results from his previous admission, which were 


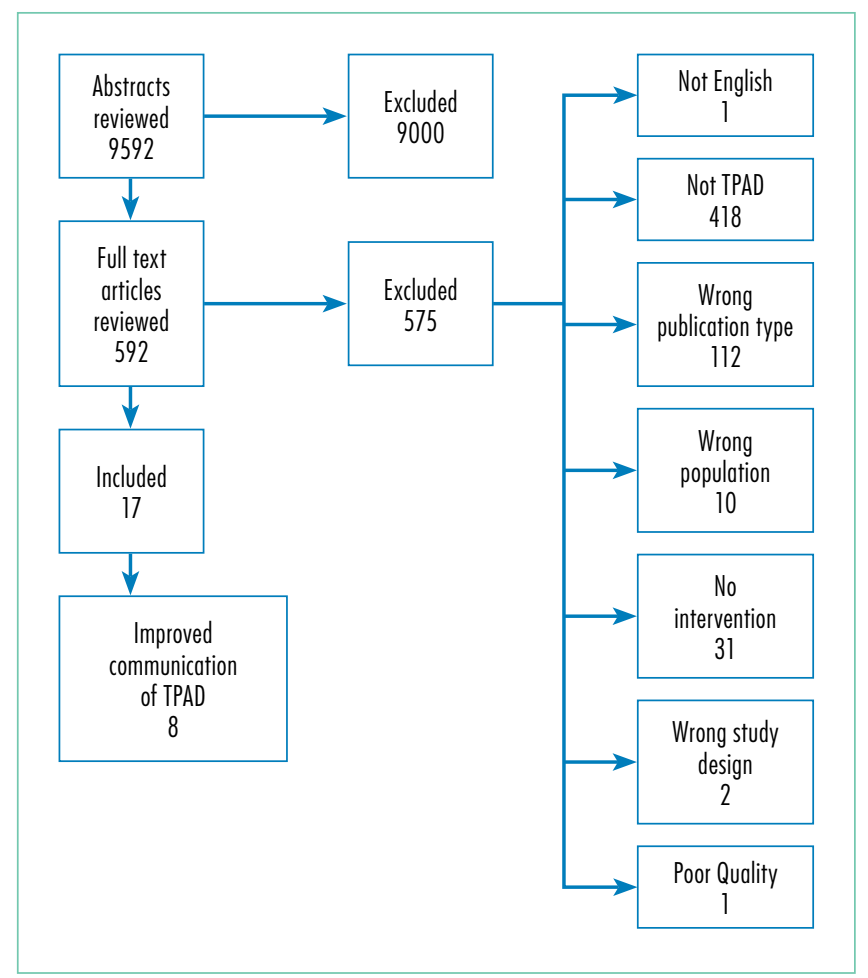

FIG 1. Literature Search Results

returned two days after his discharge, indicated that the infectious agent was not sensitive to trimethoprim-sulfamethoxazole. The results had not been reviewed by hospital clinicians or forwarded to the patient's physician, so the patient continued on the ineffective treatment. His second hospital admission lasted seven days, but he made a complete recovery with the correct antibiotic. ${ }^{10}$

Several barriers impede the follow-up of TPAD. First, who should receive test results or who is responsible for addressing them may be unclear. Second, even if responsibility is clear, communication between the provider who ordered the test and the provider responsible for follow-up may be suboptimal. ${ }^{11} \mathrm{Fi}$ nally, providers who need to follow up on abnormal results may not appreciate the urgency or significance of pending results.

The hospitalist model of care increases efficiency during hospitalization but further complicates care coordination. ${ }^{12}$ The hospitalist who orders a test may not be on duty at discharge or when test results are finalized. Primary care providers may have little contact with their patients during their admission. ${ }^{12}$ Effective communication between providers is key to ensuring appropriate follow-up care, but primary care physicians and hospital physicians communicate directly in $20 \%$ or fewer admissions. ${ }^{13}$ The hospital discharge summary is the primary method of communication with the next provider, but 65\%$84 \%$ of all discharge summaries lack information on TPAD. ${ }^{13,14}$

In this work, we sought to identify and evaluate interventions aimed at improving documentation, communication, and follow-up of TPAD. This review was conducted through the Laboratory Medicine Best Practices (LMBPTM) initiative, which is sponsored by the Centers for Disease Control and Prevention's
(CDC's) Division of Laboratory Systems (https://wwwn.cdc.gov/ labbestpractices/). The LMBPTM was initiated as the CDC's response to the IOM report To Err is Human: Building a Safer Health System. ${ }^{15}$

\section{METHODS}

We applied the first four phases of the LMBPTM_developed A-6 Cycle methodology to evaluate quality improvement practices as described below. ${ }^{16}$ Our report follows the Meta-analysis Of Observational Studies in Epidemiology (MOOSE) guidelines. ${ }^{17}$

\section{Asking the Question}

The full review, which is available from the corresponding author, assessed the evidence that the interventions improved (1) the timeliness of follow-up of TPAD or reduced adverse health events; (2) discharge planning, documentation, or communication with the outpatient care provider regarding TPAD; and (3) health outcomes. In this article, we present the impact of interventions to improve the documentation, communication, and follow-up of TPAD. The review protocol, which is also available from the corresponding author, was developed with the input of a panel of experts (Appendix A) in laboratory medicine, systematic reviews, informatics, and patient safety. The analytic framework (Appendix B) describes the scope of the review. The inclusion criteria for papers reporting on interventions to improve communication of TPAD are the following:

- Population: Patients who were admitted to an inpatient facility or who visited an ED (including patients released from the ED) and who had one or more TPADs.

- Interventions: Practices that explicitly aimed to improve the documentation, communication, or follow-up of TPAD, alone or as part of a broader quality improvement effort.

- Comparators: Standard practice, pre-intervention practice, or any other valid comparator.

- Outcomes: Documentation completeness, physician awareness of pending tests, or follow-up of TPAD.

\section{Acquire the Evidence}

A professional librarian conducted literature searches in PubMed, CINAHL, Cochrane, and EMBASE using terms that captured relevant health care settings, transition of patient care, laboratory tests, communication, and pending or missed tests (Appendix C). Citations were also identified by expert panel members and by manual searches of bibliographies of relevant studies. We included studies published in English in 2005 or later. We sought unpublished studies through expert panelists and queries to relevant professional organizations.

\section{Appraise the Studies}

Two independent reviewers evaluated each retrieved citation for inclusion. We excluded articles that (1) did not explicitly address laboratory TPAD; (2) were letters, editorials, commentaries, or abstracts; (3) did not address transition between settings; (4) did not include an intervention; (5) were case reports or case series; or (6) were not published in English. A team member 
TABLE. Summary of Evidence on Interventions to Improve Communication of Tests Pending at Discharge

\begin{tabular}{|c|c|c|c|c|c|}
\hline \multirow{3}{*}{$\begin{array}{l}\text { Education to Improve Documentation of TPAD } \\
\text { in Discharge Summaries }\end{array}$} & Dinescu (2011) & Moderate & Fair & Consistent & Suggestive \\
\hline & Gandara (2010) & Moderate & Fair & & \\
\hline & Key-Solle (2010) & Moderate & Fair & & \\
\hline $\begin{array}{l}\text { Electronic Aids to Improve Documentation of TPAD } \\
\text { in Discharge Summaries }\end{array}$ & O'Leary (2009) & Substantial & Good & & \\
\hline \multirow{2}{*}{$\begin{array}{l}\text { Electronic Notifications to Physician Responsible } \\
\text { for Follow-up }\end{array}$} & Dalal (2014) & Substantial & Fair & Consistent & Suggestive \\
\hline & El-Kareh (2012) & Moderate & Good & & \\
\hline
\end{tabular}

\section{Intervention}

Evidence that education improves completeness of TPAD information in discharge summaries

Evidence that automated tools or templates to assist in preparing discharge summary improve completeness of TPAD information

Evidence that electronic notification to the responsible physician increases their awareness of test results pending at discharge

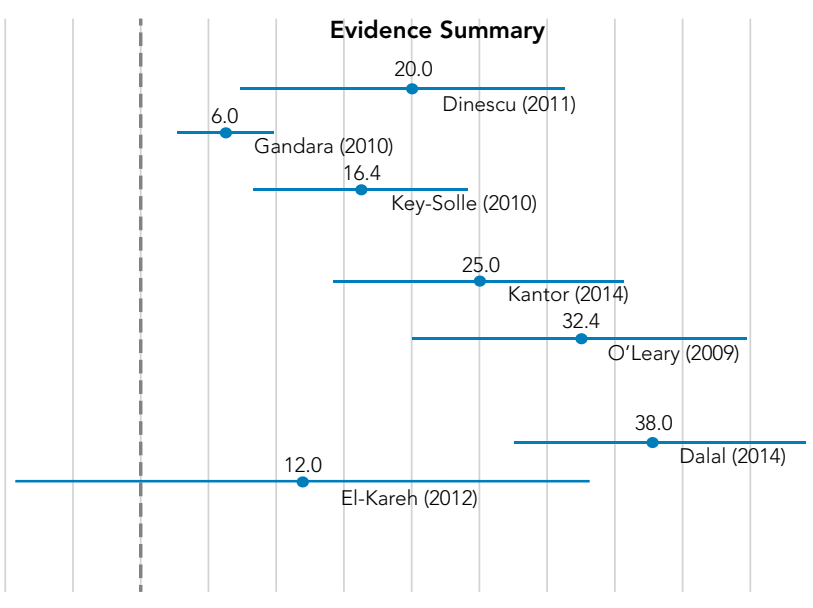

40

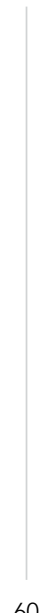

60

Does Not Support Intervention

Supports Intervention

Absolute Change in Percent

FIG 2. Impact of Interventions to Improve Communication and Follow-up of Tests Pending at Discharge

abstracted predetermined data elements (Appendix D) from each included study, and a senior scientist reviewed the abstraction. Two senior scientists independently scored the quality of the eligible studies on the A-6 domains of study characteristics, practice description, outcome measures, and results and findings; studies scored below 4 points on a 10-point scale were excluded. Based on this appraisal, studies were classified as good, fair, or poor; poor studies were excluded.

\section{Analyze the Evidence}

We synthesized the evidence by intervention type and outcome. The strength of the evidence that each intervention improved the desired outcome was rated in accordance with the A-6 methodology as high, moderate, suggestive, or insufficient based on the number of studies, the study ratings, and the consistency and magnitude of the effect size.

\section{RESULTS}

We retrieved 9,592 abstracts and included 17 articles after full-text review and study-quality appraisal; of these, eight provided evidence on communication of TPAD (Figure 1). These eight studies examined four types of interventions: (1) education to improve discharge summaries, (2) electronic tools to aid in preparation of discharge summaries, (3) electronic notification to physicians of pending tests, and (4) online access of test results for patients or parents. The Table and Figure 2 summarize the evidence for each intervention. The appendices provide detailed information on the characteristics of the included studies (Appendix E), the study interventions (Appendix F), and evidence tables (Appendix G).

\section{Education to Improve Discharge Summaries}

Three studies ${ }^{18-20}$ examined educational interventions to improve the completeness of discharge summaries, and all three 
were of fair quality with moderate effects. Two studies ${ }^{18,19}$ evaluated educational inventions for first-year residents or fellows and included individual instruction alone ${ }^{18}$ or in combination with a group session. ${ }^{19}$ Dinescu et al. ${ }^{18}$ found a $20 \%$ increase in the documentation of ordered tests, and a $39 \%$ increase in documented test results in discharge summaries (81\% vs. $42 \%$, $P=.02)$ after the intervention. Key-Solle ${ }^{19}$ reported that individual sessions resulted in a $16.4 \%(P=.004)$ increase in the documentation of pending laboratory results in the discharge summary compared with that of the controls; the group session increased documentation by only $5 \%(P=.403)$.

Gandara et al. ${ }^{20}$ conducted a multi-site, multi-intervention study to improve completeness of information in discharge summaries, including documentation of TPAD. All sites implemented physician and nurse education. A significant trend $(P<$ .001) toward more complete information overall was found after implementation; improvement in documentation of TPAD was not provided.

\section{Electronic Tools for Preparation of Discharge Sum- maries}

Two studies ${ }^{21,22}$ investigated tools to aid preparation of discharge summaries. Kantor et al., ${ }^{21}$ rated fair, evaluated an EMR-generated list of TPAD, and O'Leary et al., ${ }^{22}$ rated good, evaluated an electronic discharge summary template. The EMR-generated list resulted in an absolute increase of $25 \%$ in the proportion of TPAD documented and of $18 \%$ in the percentage of discharge summaries with complete information on TPAD. An electronic discharge summary template increased the percentage of discharge summaries with complete information on TPAD by $32.4 \% .{ }^{22} \mathrm{O}^{\prime}$ Leary et al. ${ }^{22}$ was the only study that reported a negative effect of an intervention. The authors found a 10\% $(P=.04)$ reduction in the documentation of clinically significant laboratory results after implementation of the electronic discharge summary.

\section{Electronic Notifications to Physicians}

One good study, El-Kareh et al., ${ }^{23}$ and one fair study, Dalal et al. ${ }^{24}$ examined the impact of electronic notification of pending laboratory tests or test results to physicians. El-Kareh et al. ${ }^{23}$ also provided evidence on improved follow-up of test results. Physicians in intervention clusters were three times more likely (OR 3.2; 95\% Cl 1.3-8.4) to have documented follow-up of test results than those in control clusters. ${ }^{23}$ The absolute increase in awareness of TPAD was $20 \%,{ }^{23,24}$ among primary care physicians and $12 \%{ }^{23}$ or $38 \%^{24}$ among inpatient attending physicians in the intervention clusters.

\section{Notification of Patients or Parents}

One study evaluated the impact of online parental access to the results of laboratory tests ordered during a child's ED visit. ${ }^{25}$ The intervention indirectly increased physician awareness of the test results: 36 parents (12\% of enrolled families) reported informing their physician of the test results. Therapy changed for seven children (5\% of 141 whose parents retrieved the child's test results and completed the follow-up survey).

\section{DISCUSSION}

\section{Evidence Summary}

We identified four interventions aimed at improving follow-up of TPAD and found suggestive evidence indicating that individual education for preparers of discharge summaries improved the quality of discharge summary documentation of TPAD; however, this type of evidence is below the level of evidence required by the LMBPTM to issue a recommendation. Site variations in the type and timing of interventions, ${ }^{20}$ small sample size, ${ }^{18}$ short follow-up, ${ }^{18,19}$ lack of detail on educational content, ${ }^{18-20}$ and differences in evaluated interventions limited the evidence quality. The long-term impact of educational interventions is also a concern. Oluma et al., for example, found that the benefits of education interventions were not sustained over time. ${ }^{26}$

Two studies ${ }^{21,22}$ evaluated aids to completing discharge summaries. The aids, which include a list of TPADs ${ }^{21}$ and an electronic template, ${ }^{22}$ resulted in a substantial increase in the completeness of the documentation of TPAD. Because of the differences in the interventions and the limited number of studies obtained, the evidence was rated as suggestive.

Suggestive evidence that automated e-mail notifications increased awareness of TPAD results by inpatient attending physicians and primary care providers was found. A limitation of this evidence is that both studies ${ }^{23,24}$ retrieved were conducted at the same institution; thus, the findings may not be generalizable to other institutions. Only one paper ${ }^{25}$ examined the impact of patient or parental access to laboratory tests results on the primary care physician's awareness and follow-up of TPAD; as such, we consider the available evidence insufficient to evaluate the intervention.

\section{Limitations}

The evidence regarding interventions to improve follow-up of TPAD is limited. The interventions evaluated varied considerably in design and implementation. Most studies were conducted at a single medical center. Few studies had concurrent controls, and even fewer were randomized trials. Some studies included multiple interventions, thereby rendering the isolation of the impact of any single intervention difficult to accomplish.

\section{Comparison to Other Literature}

We found no other reviews of interventions to improve follow-up of TPAD. A review of interventions to improve information transfer found that computer-generated discharge summaries improved the timeliness and, less consistently, completeness of the summary. ${ }^{13}$ The authors of this review ${ }^{13}$ recommended computer-generated structured summaries that highlight the most pertinent information for follow-up care, as supported by a recent qualitative exploration of care coordination between hospitalists and primary care physicians. ${ }^{27}$

\section{CONCLUSIONS}

Successful follow-up of TPAD during care transition is a multistep process requiring identification and documentation of TPAD, notification of person responsible for follow-up, and their recognition and execution of the appropriate follow-up 
actions. We found suggestive evidence that individual education and tools, such as automated templates or abstraction, can improve documentation of TPADs and that automated alerts to the physician responsible for follow-up can improve awareness of TPAD results. The interventions were distinct; evidence from one intervention and outcome should be applied cautiously to other interventions and outcomes.

None of the interventions completely resolved the problems of documentation, awareness, or follow-up of TPAD. New interventions should consider the barriers to coordination identified by Jones et al. ${ }^{27}$ and Callen et al. ${ }^{7}$ Both studies identified a lack of systems, policies, and practices to support communication across different settings, including lack of access or difficulty navigating electronic medical records at other institutions; unclear or varied accountability for follow-up care; and inconsistent receipt of discharge documents after initial follow-up visit. These systemic problems were exacerbated by a lack of personal relationships between the community physicians, hospital, and ED clinicians, and between acute care clinicians and patients. In EDs, high patient throughput and short length of stay were found to contribute to these barriers. Although laboratories have a responsibility, required by CLIA regulations, to ensure the accurate and complete transmission of test reports, ${ }^{28}$ none of the interventions appeared to include laboratorians as stakeholders during the design, implementation, or evaluation of the interventions. Incorporating laboratory personnel and processes into the design of follow-up solutions may increase their effectiveness.

Medical informatics tools have the potential to improve patient safety during care transitions. Unfortunately, the evidence regarding informatics interventions to improve follow-up of TPAD was limited by both the number and the quality of the published studies. In addition, better-designed studies in this area are needed. Studies of interventions to improve follow-up of TPAD need to include well-chosen comparator populations and single, well-defined interventions. Evaluation of the interventions would be strengthened if the studies measured both the targeted outcome of the intervention, such as physician awareness of TPAD, and its impact on patient outcomes. Evaluation of the generalizability of the interventions would be strengthened by multi-site studies and, where appropriate, application of the same intervention to multiple study populations. As failure to communicate or follow up on abnormal laboratory tests is a critical threat to patient safety, more research and interventions to address this problem are urgently needed.

\section{Acknowledgments}

The authors appreciate the thoughtful insights offered by the following expert panel members: Joanne Callen, PhD; Julie Gayken, MT; Eric Poon, MD; Meera Viswanathan, PhD; and David West, PhD. The authors thank Dr. Jennifer Taylor for her review of the draft manuscript.

Funding: This work was funded by contract number 200-2014-F-61251 from the Centers for Disease Control and Prevention, Division of Laboratory Systems. Dr. Singh was additionally supported by the Houston VA HSR\&D Center for Innovations in Quality, Effectiveness, and Safety (CIN 13-413).
Disclaimer: The findings and conclusions in this study are those of the authors and do not necessarily represent the official position of the Centers for Disease Control and Prevention or the Department of Veterans Affairs.

Disclosures: Drs. Whitehead, Graber, and Meleth, Ms. Kennedy, and Mr. Epner received funding for their work on this manuscript (Contract No. 200-2014F-61251) from the Centers for Disease Control and Prevention. Dr. Graber receives honoraria from several institutions for presentations on diagnostic errors and has a grant from the Macy Foundation to develop a curriculum on diagnostic errors. Unrelated to this publication, Mr. Epner receives payment as a board member of Silicon BioDevices, as a consultant to Kaiser Foundation Health Plan of Colorado, for lectures from Sysmex, Inc., and for meeting expenses from Abbott Laboratories. He has stock or stock options in Silicon BioDevices, Inc. and Viewics, Inc. No other authors have any financial conflicts to report.

\section{References}

1. National Academies of Sciences Engineering, and Medicine. Improving diagnosis in health care. 2015. http://www.nap.edu/catalog/21794/improving-diagnosis-in-health-care. Accessed January 8, 2018.

2. Schiff GD, Hasan $O$, Kim S, et al. Diagnostic error in medicine: analysis of 583 physician-reported errors. Arch Intern Med. 2009;169(20):1881-1887.

3. World Alliance for Patient Safety. Summary of the evidence on patient safety: Implications for research. Geneva, Switzerland; 2008.

4. The Joint Commission. National patient safety goals. Effective January 1, 2015. NPSG.02.03.012015

5. Poon EG, Gandhi TK, Sequist TD, Murff HJ, Karson AS, Bates DW. "I wish I had seen this test result earlier!": Dissatisfaction with test result management systems in primary care. Arch Intern Med. 2004;164(20):2223-2228.

6. Callen J, Georgiou A, Li J, Westbrook JI. The safety implications of missed test results for hospitalised patients: a systematic review. BMJ Quality Safety. 2011;20(2):194-199.

7. Callen JL, Westbrook JI, Georgiou A, Li J. Failure to follow-up test results for ambulatory patients: a systematic review. J Gen Intern Med. 2012;27(10): 1334-1348.

8. Roy $\mathrm{CL}$, Poon EG, Karson AS, et al. Patient safety concerns arising from test results that return after hospital discharge. Ann Intern Med. 2005;143(2):121-128.

9. El-Kareh R, Roy C, Brodsky G, Perencevich M, Poon EG. Incidence and predictors of microbiology results returning postdischarge and requiring follow-up. J Hosp Med. 2011;6(5):291-296.

10. Coffey C. Treatment Challenges After Discharge. WebM\&M, Cases \& Commentaries. 2010;(November 29, 2010). https://psnet.ahrq.gov/webmm/ case/227/treatment-challenges-after-discharge. Accessed November 2010.

11. Dalal AK, Schnipper JL, Poon EG, et al. Design and implementation of an automated email notification system for results of tests pending at discharge. $J$ Am Med Inform Assoc. 2012;19(4):523-528

12. Wachter RM, Goldman L. The hospitalist movement 5 years later. JAMA. 2002;287(4):487-494.

13. Kripalani S, LeFevre F, Phillips CO, Williams MV, Basaviah P, Baker DW. Deficits in communication and information transfer between hospital-based and primary care physicians: implications for patient safety and continuity of care. JAMA. 2007;297(8):831-841.

14. Were MC, Li X, Kesterson J, et al. Adequacy of hospital discharge summaries in documenting tests with pending results and outpatient follow-up providers. J Gen Intern Med. 2009;24(9):1002-1006

15. Institute of Medicine. To err is human : building a safer health system Washington, DC.1999.

16. Christenson RH, Snyder SR, Shaw CS, et al. Laboratory medicine best practices: systematic evidence review and evaluation methods for quality improvement. Clin Chem. 2011;57(6):816-825

17. Stroup DF, Berlin JA, Morton SC, et al. Meta-analysis of observational studies in epidemiology: a proposal for reporting. Meta-analysis Of Observational Studies in Epidemiology (MOOSE) group. JAMA. 2000;283(15):2008-2012.

18. Dinescu A, Fernandez H, Ross JS, Karani R. Audit and feedback: An intervention to improve discharge summary completion. J Hosp Med. 2011;6:28-32.

19. Key-Solle M, Paulk E, Bradford K, Skinner AC, Lewis MC, Shomaker K. Improving the quality of discharge communication with an educational intervention. Pediatrics. 2010;126:734-739.

20. Gandara E, Ungar J, Lee J, Chan-Macrae M, O'Malley T, Schnipper JL. Discharge documentation of patients discharged to subacute facilities: A threeyear quality improvement process across an integrated health care system. Jt Comm J Qual Patient Saf. 2010;36:243-251. 
21. Kantor MA, Evans KH, Shieh L. Pending Studies at Hospital Discharge: A Pre-post Analysis of an Electronic Medical Record Tool to Improve Communication at Hospital Discharge. J Gen Intern Med. 2014;30(3):312-318.

22. O'Leary KJ, Liebovitz DM, Feinglass J, et al. Creating a better discharge summary: improvement in quality and timeliness using an electronic discharge summary. J Hosp Med. 2009;4(4):219-225.

23. El-Kareh R, Roy C, Williams DH, Poon EG. Impact of automated alerts on follow-up of post-discharge microbiology results: a cluster randomized controlled trial. J Gen Intern Med. 2012;27:1243-1250

24. Dalal AK, Roy CL, Poon EG, et al. Impact of an automated email notification system for results of tests pending at discharge: a cluster-randomized controlled trial. J Am Med Inform Assoc. 2014;21(3):473-480.
25. Goldman RD, Antoon R, Tait G, Zimmer D, Viegas A, Mounstephen B. Culture results via the internet: $A$ novel way for communication after an emergency department visit. J Pediatr. 2005;147:221-226.

26. Olomu AB, Stommel M, Holmes-Rovner MM, et al. Is quality improvement sustainable? Findings of the American College of Cardiology's Guidelines applied in practice. Int J Qual Health Care. 2014;26(3):215-222.

27. Jones $C D, V u M B, O^{\prime}$ Donnell $C M$, et al. A failure to communicate: a qualitative exploration of care coordination between hospitalists and primary care providers around patient hospitalizations. J Gen Intern Med. 2015;30(4): 417-424.

28. Clinical Laboratory Improvement Amendments Regulations, 42 CFR 493.1291(a)(1988) 